\title{
Surveying about Different Procedures of Intensifying Cloud Security and Presenting New Idea Based on Kalman Filtering
}

Soudabeh Salehi Rekavandy Islamic Azad University of Ferdows, Department of Computer Engineering, Ferdows, Iran

Golnaz Parvaneh Hosseini Islamic Azad University of Ferdows, Department of Computer Engineering, Ferdows, Iran
Maryam Davodpour

Islamic Azad University of Ferdows, Department of Computer Engineering, Ferdows, Iran

\section{Golnaz Shahideslami} Islamic Azad University of Ferdows, Department of Computer Engineering, Ferdows, Iran
Mahdiye Abbassi Islamic Azad University of Ferdows, Department of Computer Engineering, Ferdows, Iran

\section{Hooman Kashanian}

Islamic Azad University of Ferdows, Department of Computer Engineering, Ferdows, Iran 


\begin{abstract}
Cloud is a collection encompassing a number of computers and servers that we find access to and is hosted by the internet. This technology provides capabilities for processing, storing and retrieving, support and etc. by way of the internet. Cloud computing consists of trustable services by future generation data centres that are based on virtual calculations and technologies and are provided by the internet. Now a day, numerous technologies are migrating from traditional systems to cloud. Off course, cloud can also be used as a tool for military and non-military goals. On the other hand, in this wide network where users are individuals and companies, in addition to shared use of resources, personal data is also placed in the network, need for security is very important. Frequently, users and organizations become more commonly and rapidly interested in using it when there is confidence in presence of high security methods in this service. In this paper, initially the effective role and advantages of cloud computing is evaluated in some large international military and non-military companies and organizations and ultimately security solution based on the Kalman filter is presented.
\end{abstract}

Keywords: Cloud Computing, Kalman Filter, Effect of Security in IT industry, Cloud Computing, Estimation and prediction

\section{INTRODUCTION:}

The background of computer science has persistently witnessed revolutions and fundamental changes. For example, it can be noted that in the first generation of computers that existed between the years 1945 and 1956, vacuum lamps were taken advantage of inside the computers. Later and with introduction of integrated circuits, they were used in third generation computers available in the years between 1963 and 1971 [1]. Ultimately, the fourth generation which begins from 1971 up until now alongside with development of technology large scale integration (LSI), very large scale integration (VLSI) and ultra large scale integration (ULSI) were used. Now a day also a technology named cloud computing is in the process of creating a new era in the computer industry and processing power. With study of historical trend, we can attest to the point that the idea of cloud computing sources from where processing powers can be placed in the hold of other users when current user or users do not need it [4]. The most common definition for cloud computing is access to power and processing even by way of conventional computers [7].

Examples of significant advantages of this technology are the possibility of sharing and concurrent work on a particular project or file as the following example. Assume that you what to process an individual or a special group of people's DNA [2]. If you are a cloud computing user, you will be able to first use its extraordinary stronger processing powerbecause your system might not have the necessary processing power. Second, you will be able to perform these very heavy operations concurrently with several of your friends and observe each other's work output on the project instantaneously [5]. Among its other potentials is that up to now you had to take advantage of your systems processing resources individually and once in a while you were forced to up to date your system hard and software to be able to both access the most current software versions and also had need for hard ware power for supporting your software. This by itself involves very high and in some cases unacceptable costs. In addition, if we wish to install this system on a higher number of systems, we need to purchase the license for use of the software in higher number of systems [9]. This reason contradicts the efficiency of cloud computing, because in cloud computing whenever we exit our account and reenter at another time, we witness that we will be using a newest version of the software without need to advance our systems hard ware. The reason is that the necessary hardware power has been provided by cloud. In addition, you have not paid more fees for this up to dating. In traditional systems, if you have very confidential and important information on your system, constantly due to the worry of losing or theft or manipulation of you information, you will need to update your antivirus and other security 
software (such as firewalls) [9]. If you would one day accidentally forget to perform this task, it would be possible that on the same day you would experience an attack, destruction or theft of your information. Yet, in cloud computing, you are constantly able at any time you enter your account to witness that your security system is equipped with updates made a second before. Among other advantages of cloud computing is knowledge of all suffixes. In other words, if you receive a file that is unknown in your system-since you did not know with what software to open it-with use of cloud computing, the file will be opened with the relevant software. In addition, if you own a company, you will be able to transfer your company's internal network onto the cloud computing and witness both increased speed and power and if like the past you use a server, you may not always have the amount of processing that can constantly keep the server busy and you just paid a high cost for electricity, repair and maintenance. These cases are just a corner of expansive efficiencies of cloud computing technology known as the next big thing [11].

\section{STUDY OF THE DEEP EFFECTS OF CLOUD COMPUTING ON VARIOUS INDUSTRIES}

In the prior two sections, we referred to a selection of fundamental definitions and advantages of this newly evolved technology. In this section, we will note the most major applications of this technology and with finding the sensitivity of this technology, in most of its applications, modern security strategies will be suggested by authors of this paper [6].

The major reason for using cloud computing is that humanity in the present era tends to use technologies and wireless methods of communication. Meanwhile, Amazon is the first provider of commercial cloud computing. Another reason for this amount of use is that we prefer that the servers and level of processing and other hardware not be defined and to have an unbelievable power in processing and storing and other processing operations. The third reason that can be mentioned for use of cloud computing instead of traditional systems is that now a day, humans gravitate to use of social sites. For example, the social site Facebook currently has more than 600 million registered users and everyday a huge number is added to this number. Another reason for its use is that the subscriber can decide which of its information to share with whom and in which places in the world while in traditional systems this possibility did not exist at all. Another reason is that in this method there is no longer need for separate kinds of programs, servers and systems for each group and company. Instead, each person with his or her own taste is able to use cloud computing. In addition, among other reasons we can mention lack of need for specialization in use, lack of need for a minimum level of hard and software in use and well known file formats in this system [8].

The Google Company is in the process of publishing the Chrome Factor System by way of which users can find themselves in cloud and to provide the capability of this kind of processing for them [4]. The Microsoft Company also after evaluation of this technology and the fear of losing its users is in the process of preparing Microsoft Azure which is a cloud factor system. The General Service Administration Company due to wide visits of its sites and the fear of destruction and hanging of its site decided to change hosting its site to cloud computing. National Aeronautics and Space Administration (NASA) with the NEBULA platform which is totally based on cloud computing has provided the possibility of people's participation in space projects and as a result wider possibility of storing and processing has been provided for NASA [10].

Department of Interior: this ministry which provides services for numerous federal organizations has decided to provide services based on cloud these days. Department of Health and Human Service: is in the process of proving platforms based on cloud computing for better and faster service provision. Census Bur: provider and director of SaaS services in the Salesforce site serves millions of people by way 
of cloud [9]. The White House: is in the process of transferring its system to cloud computing technology so in this way it becomes able to have automatic voting system, direct conversation with citizens and have their internal network system working faster and with increased comprehensiveness [11].

Additionally, the government of England has operationalized the wide public network of GCloud on the basis of which they can have higher precision and speed in governmental affairs. Europeans also are in the process of taking advantage of this new technology in the following governmental and public sectors: management of public sector housing, transportation service networks, census, economic development, health services and contracting and education services. Denmark has also recently with two experimental plans Digitalise'r.dk and NemHandel tested cloud computing technology for its home users and in the opinion of the Danish regional government satisfaction with the results was because of cloud computing [10].

The Japanese also with a cloud named Kasumigaseki Cloud operationalized in the Tokyo governmental-industrial locale thought of expanding cloud for use in all governmental sectors and are leaders in this area. They also have named the region and technology as a "green" environment. Of course, the latter is also a major part of the digitalization of the Japanese project [6].

China: In China also the government particularly in the northern sections of the country with a project titled "The Yellow River Delta Cloud Computing Center" is not just in the process of economic evaluation and development, but is also thinking about a government based on cloud and wider efficiency. Even the government of China in the city of Wuxi has operationalized the factory for cloud services to become enabled in providing cloud processing resources for all companies and offices [4].

Thailand: In this country GITS (Governmental Information Technology System) has designed and built a private cloud for the governmental sectors of Thailand and it is planned that soon using it various electronic services are provided for citizens and governmental sectors.

Vietnam: The IBM Company alongside with the government and universities of the country are developing and expanding the plan for cloud provision in a private and public form.

NewZealand: is in the process of expansive study on the best effects and uses possible of cloud computing [1].

Now, we will review the amazing effects of this technology on the situation of some smaller companies. A company such as the YouTube that in 2006 had 30 million pages added to its site daily should at present use cloud computing technology. The reason is that it is possible that the servers no longer have the ability for the necessary processing at time of search among all clips and films and the site of this company might face problems. Of course, if cloud computing method is not taken advantage of, servers with higher prices need to be purchased and installed which are very time consuming and costly [7].

The SmugMug Company which is a website for picture sharing for easier and increased service (such as picture conversion, retouching and etc) and also due to increased level of file volumes decided to use cloud computing. Additionally, Google provided the Google App Engine that enables you to implement your desired applications on Google substructures. In this way, you are able to share the documents you wish with specific people or in general at a global level. This service is totally free by the IBM company and Google is in the process of operationalizing a network on the basis of these new calculations for universities so via it, universities can perform their activities in a more focused and stronger way and if students of other universities have ideas or opinions about the active project, they can have a share in it. The first universities that connected to this network are University of Washington, Carnegie-Mellon University, Stanford University, The University of California at Berkeley and Maryland University [3].

The NASDAQ Company that has a wide stock and fund data base has decided to share and sell 
them. Yet, their company was concerned with server use and level of their space occupation and therefore, it used the S3 service of Amazon. Additionally, smaller companies such as Nimbus and Eucalyptus are recently providing computational, storing and applied services with a fee [2].

The Sun Micro Systems company is in the process of operationalizing modern data centers for hosting cloud computing applications and users. This company in various locations globally has provided sites with powerful servers for support so potential defects can also be avoided [5].

Networks such as My experiment (www.myexperiment.org) and nanoHub (www.nanohub.org) currently are in the process of migrating to cloud computing for sharing results of research and investigational works with people and direct connection with them.

Additionally, cloud computing provides banking services such that you can gain access to services with payment of a fee and will be able to perform tasks to acquire a payment from other users or improve a service and sell it and overall a buy and sale system will be accomplished [7]. Ultimately, the authors of this paper predict that in a future not very far, numerous companies and individuals will be occupied with provision of cloud computing services and technologies. Technicians and designers should also perform their role in the development and expansion of cloud computing correctly and provide services in accord with user need, simplify their access, provide more appropriate structure, higher capacity and capability and the possibility of adding and omitting resources [2].

\section{NEW SECURITY STRATEGY SUGGESTED BY THE AUTHORS:}

In the first and second sections, the principles of cloud computing and some wide advantages were evaluated. In the third section, application of cloud computing in some of the major companies and sectors was evaluated and analyzed carefully. Now, it is time for authors of the paper to introduce the new security idea.
As you know, many companies face weakness in processing power because they do not have the ability to use very powerful servers and computers-for example due to economic reasons. Also, if they have such powerful systems, they will not have the kind of processing that can constantly engage these systems and they will bear an extra cost of repair and maintenance. Therefore, the only suggestions at present to them is that cloud computing is used. Yet, when we suggest to them to use cloud computing, we need to give companies and organizations particularly public and military institutes peace of mind when it comes to security. On this basis, the following idea is presented [3]:

The Kalman filter is an effective recursive filter that estimates the situation of a dynamic system from a sequence of defected and confounded evaluations. An example of the application of this filter is presentation of precise and up to date data persistently regarding place and speed of a hypothesized object only by having a sequence of observations from its location. Each of these observations comes along with a small error. This filter is used in a wide domain of engineering applications from radar to computer visualization and is an important topic in the theory of control and engineering of control systems. Now a day, broad composites of Kalman filters have expanded: From initial formulas-which are now called simple Kalman filters- to Smith's generalized filter-information filter- and a range of root of squares filters that have been designed by Bayerman, Torenton and many others.

Unlike other estimation filters such as the Wiener1 (that can only be implemented on static systems and cannot include effects of preliminary conditions), Kalman filters can be implemented on both static and non static processes and include the preliminary conditions of processes in estimation, prediction, filtering or best accidental control algorithms.

\section{CONCLUSION:}

The authors suggest if we equip the cloud computing system with the Kalman filter, we 
will be able to perform the necessary prediction of level of needed resources, number of online users in a particular hour and tracking an intrusive individual in the network easily. The reason is that exactly like rocket prediction in this section also with attention to the kind of movements of the hacker and his or her movement analysis; we will be able to easily track and expose him or her.

\section{REFERENCES}

1. The NIST Definition of Cloud Computing". National Institute of Standards and Technology. Retrieved 24 July 2011.

2. "What is Cloud Computing?". Amazon Web Services. 2013-03-19. Retrieved 2013-03-20.

3. Oestreich, Ken, (2010-11-15). "Converged Infrastructure". CTO Forum. Thectoforum.com. Retrieved 2011-12-02.

4. Schmidt, Eric; Rosenberg, Jonathan (2014). How Google Works. Grand Central Publishing. p. 11. ISBN 978-1-4555-6059-2.

5. Rochwerger, B.; Breitgand, D.; Levy, E.; Galis, A.; Nagin, K.; Llorente, I. M.; Montero, R.; Wolfsthal, Y.; Elmroth, E.; Caceres, J.; BenYehuda, M.; Emmerich, W.; Galan, F. "The Reservoir model and architecture for open federated cloud computing". IBM Journal of Research and Development 53 (4): 4:1-4:11.

6. Kyriazis, D; A Menychtas; G Kousiouris; K Oberle; $T$ Voith; $M$ Boniface; E Oliveros; $T$ Cucinotta; S Berger (November 2010). "A Realtime Service Oriented Infrastructure". International Conference on Real-Time and Embedded Systems (RTES 2010) (Singapore).

7. Dario Bruneo, Salvatore Distefano, Francesco Longo, Antonio Puliafito, Marco Scarpa: Workload-Based Software Rejuvenation in Cloud Systems. IEEE Trans. Computers 62(6): 1072-1085 (2013)

8. Voorsluys, William; Broberg, James; Buyya, Rajkumar (February 2011). "Introduction to Cloud Computing". In R. Buyya, J. Broberg, A.Goscinski.

9. Alcaraz Calero, Jose M.; König, Benjamin; Kirschnick, Johannes (2012). "Cross-Layer
Monitoring in Cloud Computing". In Rashvand, Habib F.; Kavian, Yousef S.

10. Amies, Alex; Sluiman, Harm; Tong, Qiang Guo; Liu, Guo Ning (July 2012). "Infrastructure as a Service Cloud Concepts".

11. Metzler, Jim; Taylor, Steve. (2010-08-23) "Cloud computing: Reality vs. fiction," Network World. 